\title{
Tecnura
}

\section{Evaluación cualitativa de la utilización del lenguaje de programación visual kodu en niños de educación básica}

\author{
Qualitative assessment of the kodu visual programming language \\ in primary school children
}

\author{
Sir Alexci Suarez Castillón¹, Fanny Stella Soto Arévalo²
}

Fecha de recepción: 6 de abril de 2015

Fecha de aceptación: 24 de agosto de 2015

Como citar: Suarez Castillón, S. A., \& Soto Arévalo, F. S. (2015). Evaluación cualitativa de la utilización del lenguaje de programación visual KODU en niños de educación básica. Revista Tecnura, 19(46), 37-48. doi:10.14483/ udistrital.jour.tecnura.2015.4.a03

\section{Resumen}

El objetivo de este artículo es evaluar el pensamiento científico de los niños mediante el lenguaje de programación KODU, basado en la iniciativa de resolución de problemas, pensamiento crítico e innovación natural que poseen. Para ello se utiliza un proceso enmarcado dentro del club de ciencia denominado La Universidad de los Niños, compuesto por seis fases y cuatro roles, como son: diseñador, programador, test y cliente. Los resultados demuestran que los niños tienen un conocimiento sobre las propiedades de los objetos y su mundo real y virtual, lo que les permite desarrollar una programación orientada a objetos, sin los conocimientos previos de este paradigma. Las pruebas han demostrado que pueden tomar decisiones por medio de la investigación, trabajar en un ambiente colaborativo, corregir sus errores y tener un pensamiento científico que les permiten atreverse a innovar en todo momento. Los resultados demuestran que más del $90 \%$ de los niños ha respondido satisfactoriamente a los retos planteados mediante el lenguaje de programación, lo que permite darles una idea del trabajo de un ingeniero de sistemas.
Palabras clave: KODU, lenguajes de programación, pensamiento científico, programación visual, Universidad de los Niños.

\begin{abstract}
This article is aimed to developing scientific thinking in children from the perspective of Systems Engineering program through KODU Visual programming language. This is based on the ability children have to resolving problems, critical thinking, and their natural inborn talent. In order to accomplish this task, the process is part of a science club named University of the Children. This is a project with six different phases and four roles to be played as a designer, a programmer, a testing person, and a client. The results show that children have knowledge on the properties of objects, and about their real and virtual world, which allows them to develop a language program focused on objects without having a prior knowledge on this paradigm. It has been demonstrated by proof that children can make decisions by doing research and working with peers. They can also develop scientific knowledge and correct mistakes, and that leads them to dare to be innovative at all times. The results show that more
\end{abstract}

\footnotetext{
Ingeniero de sistemas, doctor en Sistemas Inteligentes en la Ingeniería. Docente Auxiliar, Universidad Francisco de Paula Santander Ocaña, Departamento de Ingeniería de Sistemas e Informática. Ocaña, Colombia. Contacto: sasuarezc@ufpso.edu.co

2 Contadora pública, Msc(c) Administración de las Organizaciones. Docente de cátedra, Universidad Francisco de Paula Santander Ocaña. Ocaña, Colombia. Contacto: fssotoa@ufpso.edu.co
} 
than $90 \%$ of children have responded with satisfaction to the challenges proposed by programming language which gives them a better idea of the role, functions and responsibilities of a systems engineer.
Keywords: KODU, Program languages, Scientific thinking, University of the Children, Visual programming.

\section{INTRODUCCIÓN}

El rol de la universidad como motor de desarrollo social y como creadora de conocimiento, cultura y pensamiento debe despertar vocaciones científicas mediante herramientas innovadoras para facilitar el trabajo didáctico en las instituciones educativas, y favorecer la interacción entre la universidad, las instituciones de educación y las familias.

Uno de los inconvenientes en la educación superior es la gran deserción que existe en las carreras de ingenierías y el poco interés en la investigación científica, a causa de que muchos estudiantes han perdido su autonomía e interés y razonamiento abstracto y lógico, debido a una enseñanza con modelos muy estrictos y basados en la memorización y repetición de lo que dicen los libros.

Partimos de la base de que los niños y niñas tienen un pensamiento científico y un gran interés por la ciencias en sus inicios escolares (Gallego, Castro, \& Rey, 2008; Merzagora \& Jenkins, 2013) , sin embargo van disminuyendo su compromiso y formas de resolver los problemas en la medida en que avanzan en su vida escolar, debido a problemáticas familiares y modelos pedagógicos implantados por docentes que coartan la curiosidad, la iniciativa y la búsqueda de soluciones innovadoras. Los niños son imitadores a cada momento (Buchsbaum, Gopnik, \& Griffiths, 2010); seleccionan la mejor opción que les permita resolver sus inquietudes, pero científicamente terminan seleccionando la opción que más se repita, sin importar su conveniencia, por lo tanto los procesos que inculcan diariamente los docentes hacen que su pensamiento e ideas se conviertan en un patrón repetitivo. Una sus consecuencias es la pérdida de capacidades estadísticas, las cuales se ven reflejadas en los deseos, preferencias, creencias, emociones y estados de conocimiento que les permite estudiar y entender al ser humano algo muy semejante a la función que ejerce un sicólogo con todos sus instrumentos de medición, (Kushnir, Xu, \& Wellman, 2010; Lukas, Griffiths, Fawcett, \& Gopnik, 2014; Ma \& Xu, 2014).

Recientes investigaciones apuntan a solucionar este tipo de conflicto que algunas veces puede ser generado en los niños y niñas, y que permiten que en algunos casos aprendan mejor que los adultos. En uno de estos experimentos (Lucas, Bridgers, Griffithsc, \& Gopnik, 2014) se comprobó que los niños aprenden las relaciones causales con rapidez. La prueba fue realizada con niños de entre 4 y 5 años de edad, de un total de 32 chicos y 143 adultos.

La metodología se basó en un conjunto de eventos los cuales se dividían en una prueba de entrenamiento, que a su vez estaban divididas en diferentes objetos(por ejemplo, el objeto A era un triángulo, el objeto $\mathrm{B}$ era un círculo, el objeto $\mathrm{C}$ era un cubo). El entrenamiento consistía en fusionar dos objetos, en uno por ejemplo se fusionaba el triángulo con el círculo y el resultado era una pirámide, mientras que en los eventos de prueba ya se encontraban los objetos finales como una pirámide, la estrella, etc. O sea que el experimento consistía en mostrar los objetos finales o de prueba y decirles a los niños y adultos que intentaran fusionar los objetos de la prueba de entrenamiento que formaran el objeto final. Los resultados demostraron que los niños acertaban casi en un $90 \%$, mientras que los adultos solo llegaban a un $71 \%$.

Varios investigadores han establecido una estrategia metodológica innovadora dirigida a la enseñanza de la ciencia, donde se destaca la participación del docente y el entorno familiar, se utilizan materiales y equipos de uso cotidiano, y se 
plantea la importancia del inicio temprano en la formación en distintas áreas (Trujillo, 2001). En el trabajo citado se realiza un experimento donde los niños sacan conclusiones o planifican sus acciones con base en impresiones físicas inmediatas, es decir que según las características de un objeto, ellos lo pueden clasificar según la presencia o ausencia de una característica mediante comparaciones, y logran representar sus descubrimientos de una manera sencilla. Esto prueba que los niños desarrollan sus habilidades mentales siempre que estos objetos se puedan manipular físicamente.

Otros investigadores han utilizado herramientas que consoliden y permitan un pensamiento innovador a edades tempranas, como son el acceso a las nuevas tecnologías, que les ayuda a despertar un verdadero interés por la informática (Barrionuevo, 2009). Si partimos desde la función principal de un computador, la cual es solucionar problemas, también debemos entender que por sí solo un computador no hace nada si no cuenta con las instrucciones necesarias para ejecutar un programa. Esto se logra por medio de órdenes que deben ser pensadas, diseñadas, desarrolladas y finalmente implantadas para su posterior uso o prueba, tal proceso implica una clara reestructuración de todos los componentes y ayuda a generar preguntas y pensamientos innovadores en los niños, los cuales permiten consolidar un pensamiento científico que abarque la investigación del problema a resolver.

Uno de estos programas es el software de videojuegos KODU, el cual permite una programación orientada a objetos de forma visual (Miraut \& Mendoza, 2011; Cacia, Chiazzese, \& D' Amicoa, 2013), de tal forma que se puede programar de forma intuitiva, así los mismos usuarios programan sus tareas sin contar con un conocimiento técnico, ni reglas predeterminadas. Además de KODU existen otros paquetes informáticos como Smalltack, Squeak, Scratch, Plopp, Alice, etc., que ayudan a incentivar la informática o al menos a conocer el mundo de los bits y la labor que desempeñan los ingenieros de sistemas.
Aunque ya existen estudios donde utilizan herramientas informáticas que permiten al niño pensar de una manera más libre, no hay un modelo que los incentive a seleccionar o conocer las áreas de ingeniería; es por eso que en este artículo se pretende plantear la curiosidad y despertar el interés por la investigación y la lógica, con base en el proyecto denominado La Universidad de los Niños, específicamente en uno de sus componentes, el niño Ingeniero de Sistemas, implementado mediante un proceso basado en roles por medio del software de videojuegos KODU, con el propósito de fortalecer el pensamiento analítico, sistémico y creativo.

\section{METODOLOGÍA}

La investigación se basa en un conjunto de pruebas que abarca la creación de roles, historias y mundos virtuales, y da a conocer al niño una idea central de la profesión de ingeniero de sistemas, de tal forma que pueda afianzar sus conocimientos y se generen nuevos enfoques en la solución de los problemas que plantea la historia, basados en un pensamiento científico, a la vez que puede ser para eliminar los viejos paradigmas de la programación.

\section{Modelo de niño Ingeniero de Sistemas}

El niño Ingeniero de Sistemas es un componente del proyecto institucional La Universidad de los Niños, el cual pretende acercar la universidad a los niños y niñas, así como a los adolescentes y a sus familias. Es una experiencia inédita en La Universidad Francisco de Paula Santander Ocaña (UFPSO), que nace desde cada una de sus carreras y su área de formación. A través esta propuesta, los niños pueden tener un contacto con la ciencia, la tecnología y la cultura.

La primera Universidad de los Niños y las Niñas tuvo lugar en la Universidad de Tübingen (Alemania) en 2002: fue una iniciativa de los periodistas Ulrich Janssen y Ulla Steuernagel, quienes, 
posteriormente, recogieron el contenido de las charlas impartidas en unos libros que se publican periódicamente. Desde entonces, la idea se ha extendido por todo el mundo y actualmente hay más de 200 Universidades de los Niños y las Niñas en toda Europa, y algunas más en América Latina y Canadá. Como resultado del éxito de la iniciativa se ha creado la European Children's Universities Network.

La diferencia de este proyecto con esta red es que el proyecto institucional se enfoca en "minicarreras", de tal forma que cada niño pueda ir recorriendo cada una y se incline por la que sienta más afinidad. El proyecto contiene tres ejes estratégicos: a) Los niños descubren la Universidad; b) Apoyando las decisiones del futuro y c) Apoyo a la investigación; dentro de las actividades y grados que se imparten figuran, entre otras: niño Ingeniero de Sistemas, niño Ingeniero Civil, niño Contador Público, niño Comunicador Social.

No poder aprender conocimientos de informática puede entorpecer la habilidad de un estudiante para ingresar a la universidad o para conseguir un trabajo después de graduarse de la escuela secundaria. Por esto se crea el niño Ingeniero de Sistemas. Dentro de los objetivos que se pretenden obtener se encuentran: a) Motivar a los niños enseñándoles a resolver problemas de forma creativa así como el arte de programar; b) Involucrar a los niños con la ciencia y la universidad de una manera diferente, promoviendo el acompañamiento científico que el docente necesita; c) Brindar los conocimientos necesarios para comprender la labor del ingeniero de sistemas en un ambiente real, creativo y lúdico; d) Incentivar a los niños a seguir en un proceso de formación continua; e) Permitir a los niños que sigan pensando como verdaderos científicos.

\section{Población, herramientas y roles informáticos}

La muestra consistió en 17 estudiantes con edades de 7 a 9 años, con cuatro roles basados en ingeniería de sistemas. La solución al problema consistía en la creación de una historia narrativa, titulada El perro náufrago, y posteriormente se debía encontrar la mejor solución mediante la creación de un videojuego. Los pasos consistían en:

- Un conjunto de lecciones, planes y actividades que permiten al niño comprender y realizar las labores de un ingeniero de sistemas centrado en una herramienta de programación visual.

- Para producir el videojuego se debe tener un conjunto de colaboradores con roles que permitan crear una historia, diseñarla y realizarla. Por eso los roles que cada niño o niña deben tomar son: guionista, diseñador, programador y probador o test.

- Para realizar esta labor, el guionista debe proponer una historia, que el diseñador plasmará mediante recortes de objetos en una pizarra de papel, los cuales debe seleccionar de un conjunto de objetos; posteriormente, el programador decide las funciones y movimientos de cada objeto, mediante líneas de dirección dibujadas en la misma pizarra. Una vez se plasma toda la historia en la pizarra, se utiliza el software KODU, lo que permite llevar a la realidad el bosquejo de la historia y por consiguiente el videojuego.

- Para lograr este objetivo un docente o estudiante de un semillero enseña y guía al niño en el manejo del software, lo que permite la realización y prueba del videojuego.

\section{Desarrollo de programación}

El proceso contiene seis fases de máximo trienta minutos cada una (tabla 1), compuestos por cuatro integrantes, quienes crearán una empresa que les permita generar el mejor videojuego para su venta final al docente líder de la actividad. Así que el niño Ingeniero de Sistemas inicia con una presentación del video "¿Qué es un ingeniero de sistemas?", donde muestran las labores del ingeniero de sistemas y la importancia de sus funciones en una empresa.

Posteriormente se presenta un videojuego realizado en KODU para lograr que el niño comprenda 
Tabla 1. Fases de desarrollo de la actividad

\begin{tabular}{ll}
\hline \multicolumn{1}{c}{ Fases } & \multicolumn{1}{c}{ Beneficio } \\
\hline $\begin{array}{l}\text { Presentación del video “¿Qué es un Ingeniero } \\
\text { de Sistemas?" }\end{array}$ & $\begin{array}{l}\text { Entender las labores del ingeniero de sistemas y la importancia de sus fun- } \\
\text { ciones en una empresa. }\end{array}$ \\
\hline $\begin{array}{l}\text { Presentación de un videojuego realizado en } \\
\text { KODU }\end{array}$ & $\begin{array}{l}\text { Lograr que el niño comprenda la problemática planteada y cómo debe } \\
\text { quedar el proyecto final. }\end{array}$ \\
\hline Presentación de la historia El perro náufrago & $\begin{array}{l}\text { Comprender la historia y el guión a seguir para desarrollar un videojuego } \\
\text { con base en diferentes etapas. }\end{array}$ \\
\hline $\begin{array}{l}\text { Desarrollo y solución de las diferentes etapas } \\
\text { del videojuego utilizando la pizarra de papel }\end{array}$ & $\begin{array}{l}\text { Aprender a entender los problemas planteados, generando diferentes solu- } \\
\text { ciones con una retroalimentación constante. }\end{array}$ \\
\hline $\begin{array}{l}\text { Codificación y prueba del videojuego mediante } \\
\text { KODU }\end{array}$ & $\begin{array}{l}\text { Aprendizaje y experiencia mediante la programación visual. De esta } \\
\text { manera se sentirán en un ambiente agradable dentro de una empresa de } \\
\text { ingeniería de sistemas. }\end{array}$ \\
\hline Concurso mejor videojuego & $\begin{array}{l}\text { Entender que dentro de una empresa debe existir responsabilidad, justicia y } \\
\text { compromiso. }\end{array}$ \\
\hline Rueda de prensa & \begin{tabular}{l} 
Conocer cuánto aprendieron y cómo quieren innovar. \\
\hline
\end{tabular}
\end{tabular}

Fuente: Elaboración propia.

la problemática planteada y cómo debe quedar el proyecto final; en este paso se dan unas explicaciones simples de programación visual de KODU, las cuales abarcan la creación de un mundo (figura 1), creación de objetos, correspondencia de puntajes, creación de obstáculos y movimientos simples. Es de destacar que el software KODU es gratuito y puede ser utilizado con alumnos que presentan dificultades de concentración o de compresión, o hacer más interesante las clases de informática o lógica.

El software contiene diferentes plantillas con mundos ya creados y objetos con propiedades de movimientos; sin embargo, al crear la historia de forma narrativa y brindar la posibilidad de crear su propio videojuego, se inicia con un mundo virtual vacío, el cual contiene todas las propiedades para programar el videojuego (figura 2).

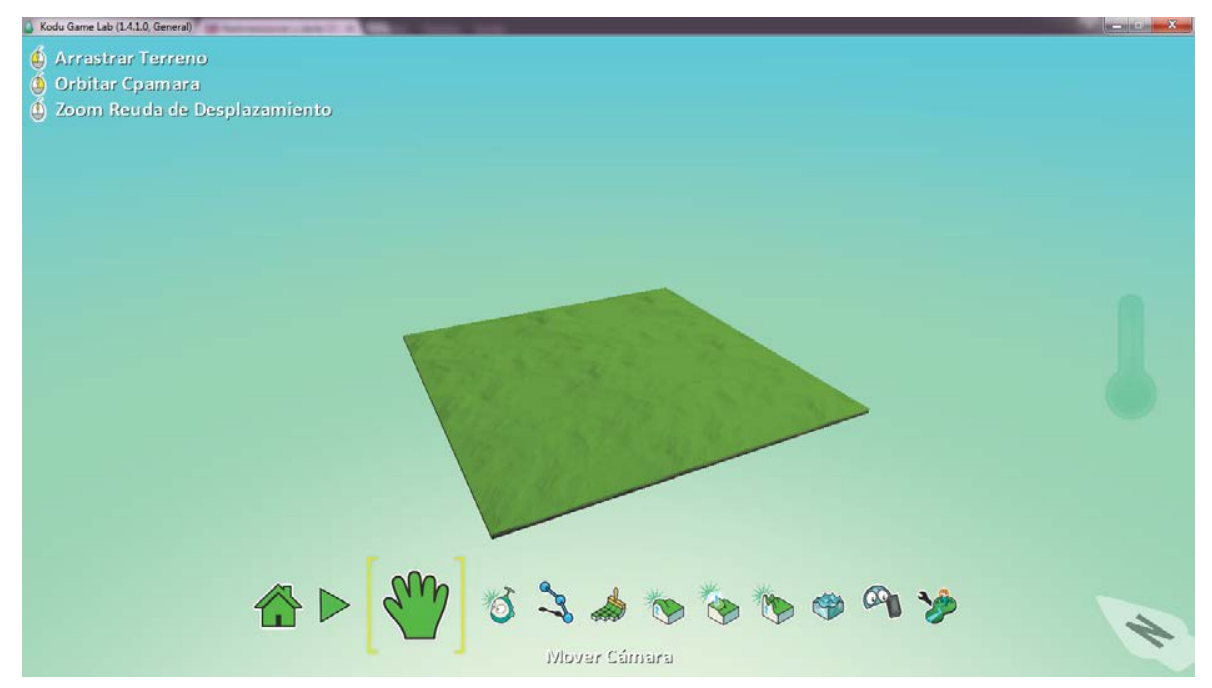

Figura 1. Creación del mundo.

Fuente: Software KODU. 


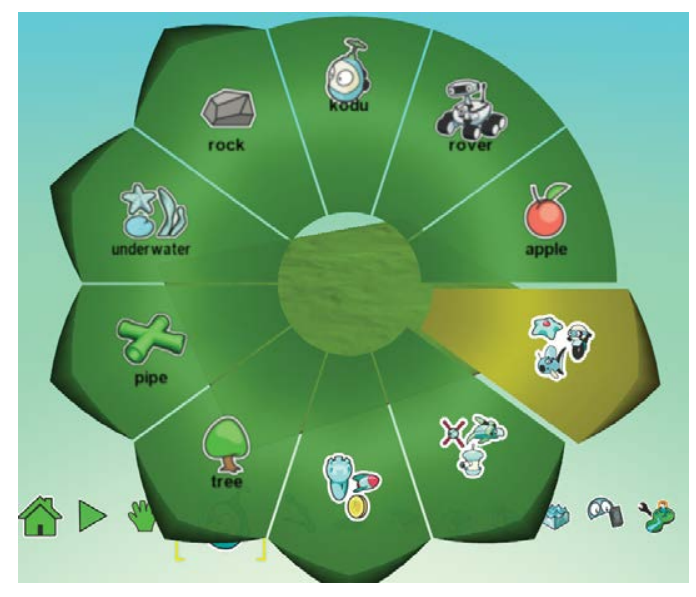

Figura 2. Panel de creación de objetos.

Fuente: Software KODU.

La prueba final es la realización de la historia en un videojuego, para lo cual cada grupo de niños que formó una empresa, se dispone con un estudiante integrante de un semillero de investigación quien empieza a mostrarles la forma de diseñarlo y darle movimiento. Es aquí donde empieza un refactoring y donde encuentran una metodología que les permite aumentar o reducir las funciones de los objetos con la finalidad de encontrar la mejor solución.

En la figura 3 se puede observar la creación de diferentes objetos, los cuales permiten crear el mundo de una manera muy intuitiva, tan solo al seleccionar el objeto del panel y dando un clic en

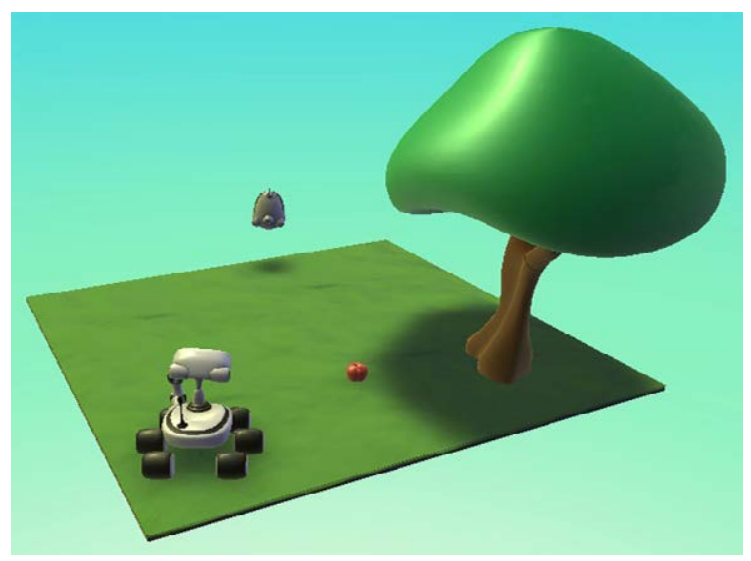

la posición donde debe insertarse; y al presionar sobre un objeto y darle clic derecho, aparecen las diferentes propiedades, de tal forma que se pueda programar o cambiar el diseño.

La programación se realiza por medio de líneas visuales, donde para darle movimiento al objeto se selecciona el teclado y la imagen de flechas; para moverlo se selecciona el objeto; si se quiere dar un puntaje, se encuentran opciones como set score o points, así que cada objeto tiene un abanico de posibilidades (figura 4).

Después de conocer el software se presenta la historia, basada en un cachorro que se encuentra distante de su casa, por lo que deben crear un mundo que lo lleve por diferentes lugares, rutas y formas de alimentación. El propósito central es que el cachorro pueda llegar a su destino, de tal forma que se presenta una maqueta donde están el cachorro (en la posición izquierda inferior del plano) y su casa (posición derecha superior). Para llegar allí debe encontrar la ruta adecuada, tomando en cuenta que si no se alimenta puede morir en el camino o si se alimenta mal puede envenenarse. Después de presentar la historia, cada niño empieza a seleccionar los roles de ingeniero. Cada rol tiene una función muy importante: el guionista es el encargado de redactar la historia, y funciona de una manera similar a un cliente, debido a que dará las instrucciones y solicitará el desarrollo del programa con base en sus

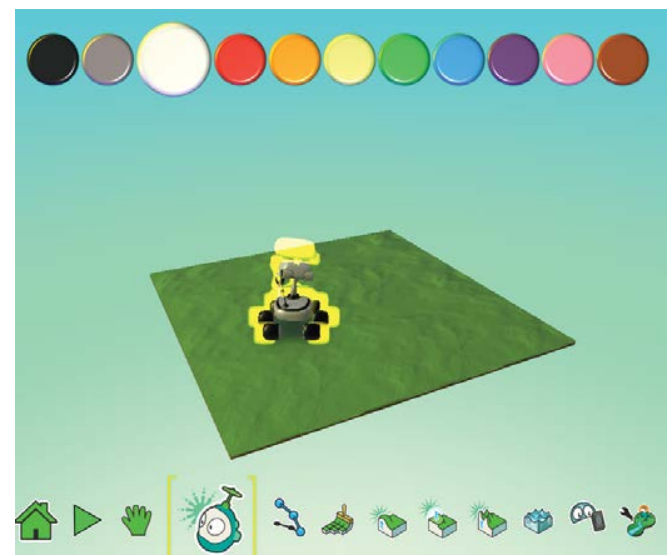

Figura 3. (a) Creación de objetos; (b) Menú de diseño y programación.

Fuente: Software KODU. 


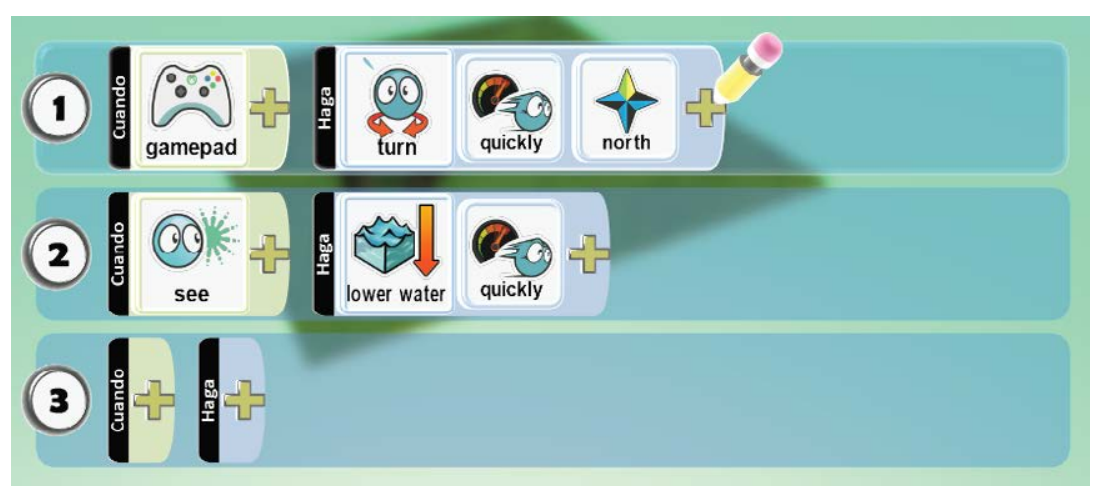

Figura 4. Programación de objetos.

Fuente: Software KODU.

necesidades; el diseñador es el encargado de formar las imágenes de los objetos, las cuales se encuentran en plantillas de papel que son recortadas y pegadas con una idea lógica que permitan al programador desarrollar el recorrido, darle nombre a los objetos y valor de premiación o castigo; finalmente el probador se encarga de comprobar que la historia y la programación sean correctas. Al final, los niños son los encargados de calificar el trabajo de sus compañeros dándoles una puntuación de 1 a 5 .

\section{RESULTADOS}

\section{Experimento (fase I, II, III)}

La fase uno se inicia con una pregunta cotidiana sobre si conocían la función del ingeniero de sistemas, que se volvía a repetir después de mostrarles el video (tabla 2).

Se pudo comprobar que el niño capta la información del video y se hace a una idea global de las funciones del ingeniero de sistemas, donde se cambia la visión del mecánico o del que arregla computadores, y se le da importancia a los que realizan las pruebas finales. En la fase dos se mostró un videojuego realizado en KODU con la intención de saber si tenían una percepción del software y del mundo virtual, así como del objetivo que debían plantearse, con obstáculos, puntos y objetos (tabla 3 ).

En la fase tres se presentó la historia del pero náufrago y los objetivos que debía cumplir el cachorro para llegar a su destino. Es aquí donde el niño empieza a desarrollar su pensamiento

Tabla 2. Conocimiento de la carrera de ingeniería de sistemas

\begin{tabular}{lccccc}
\hline \multicolumn{1}{c}{ Proceso } & Programador & Maneja computadores & Diseña & Prueba & Repara \\
\hline Inicio fase & 1 & 11 & 0 & 0 & 5 \\
\hline Fin fase & 8 & 0 & 4 & 3 & 0 \\
\hline
\end{tabular}

Fuente: Elaboración propia.

Tabla 3. Percepción del software.

\begin{tabular}{cccccc}
\hline Número & Objetos & Puntos & Movimiento & Colores & Rutas \\
\hline Cantidad & 15 & 11 & 8 & 5 & 4 \\
\hline
\end{tabular}

Fuente: elaboración propia. 
innovador y a proponer ideas y realizar preguntas al docente. Una de estas preguntas estaba relacionada con que en la historia presentada no había un premio para el cachorro al llegar a su casa, y también que si el cachorro comía frutas podía morir o simplemente descontarle puntos. En esta fase se observaron las iniciativas propias de cada niño, individualmente, por medio de preguntas como:

- Se detectaron niños líderes en el proceso (A1).

- El niño buscaba diferentes tipos de soluciones (A2).

- Entendieron que las láminas impresas con figuras eran los objetos para realizar la historia y finalmente el programa (A3).

La manera de saber si los niños tenían la solución a cada pregunta se hacía por medio de la respuesta oral o al levantar la mano como afirmación. Para la pregunta A1, la cantidad de niños que respondieron fueron 5 ; para la A2, 12; y 6 para la A3. Las respuestas obtenidas daban una idea global de la solución por parte de cada niño, sin la orientación del docente, ya que implicaban diferentes estrategias para el desarrollo de la historia y posterior programación.

\section{Experimento (fase IV)}

Esta fase estudiaba el liderazgo de cada niño mediante la selección del rol a utilizar. Existían cuatro grupos creados, dos de cuatro y dos de cinco; se les preguntó a cada uno el rol que quería tomar. Los resultados son los siguientes:

Como se puede observar en la figura 5, los niños se inclinaban más por el rol de diseñador en la pizarra de papel, ya que podían reorientar la historia, diseñar y dar instrucciones para la resolución del problema, de forma que utilizaban todas sus destrezas, algo que no sucedía a la hora de elegir el computador, donde se orientaban por el rol de programador y probador, debido a que estos roles eran los más importantes y podían tomar el control de la situación, imponer sus ideas y rediseñar el juego mediante los comandos de programación.

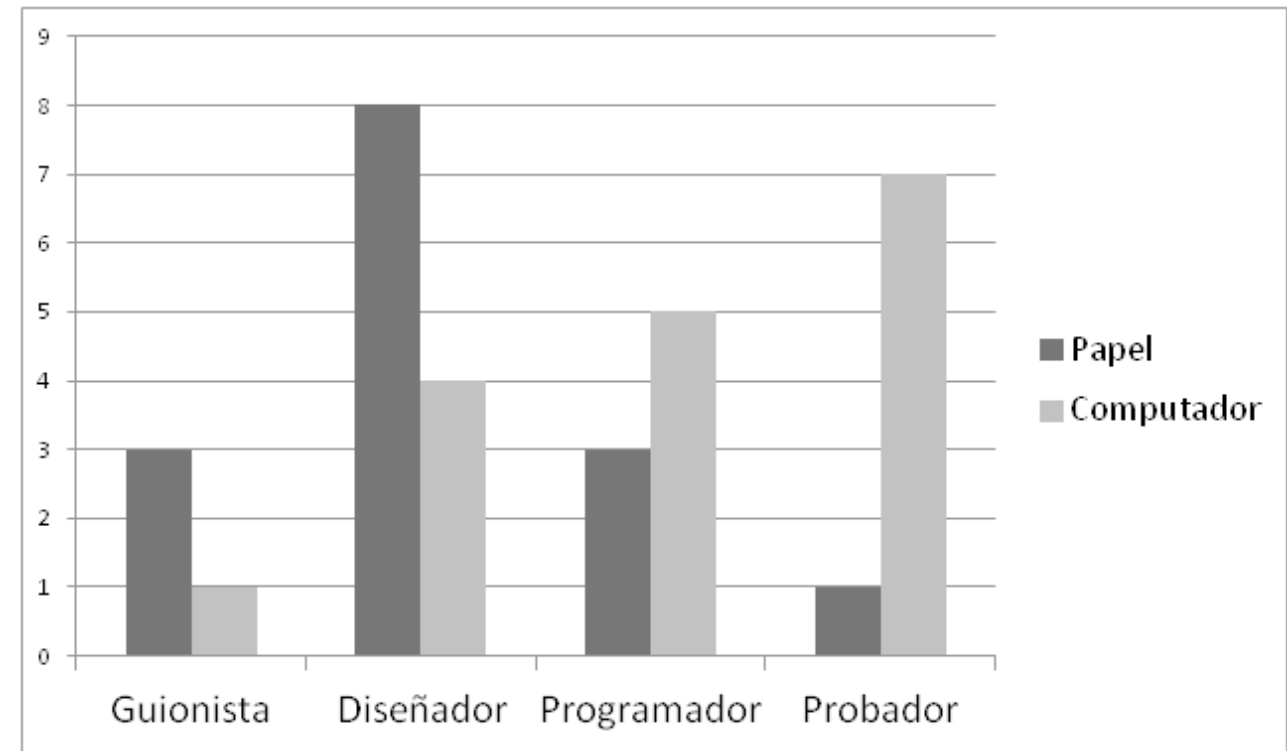

Figura 5. Diferencia entre los roles seleccionados en papel y KODU

Fuente: Elaboración propia. 
Existe una gran diferencia entre el rol de probador seleccionado en la pizarra de papel y el computador, por eso la selección de este rol en el computador indica que existen más niños que se adelantan a la solución del problema y tienen diferentes técnicas que permiten probar el resultado final del juego, de tal forma que daban opiniones a los diseñadores y programadores, sin necesidad de cambiar la historia creada por el guionista; inclusive tenían la habilidad para tomar el control de los dos roles anteriores.

\section{Experimento (fase V, VI)}

Esta fase permite el aprendizaje mediante la programación visual de KODU, de esta manera se sentirán en un ambiente agradable dentro de una empresa de Ingeniería de Sistemas, y desarrollaron todas sus habilidades, sin restricciones. El lenguaje KODU es utilizado para resolver problemas de índole matemático y estrategias de competencia; por eso, en la fase $V$ se planteaban dos estrategias: la primera era la forma como se diseñaba el juego y la segunda, la forma de utilizar los elementos creados en él para superar el reto. Estas dos estrategias permiten demostrar que los niños mantienen un pensamiento científico enfocado a la resolución de problemas, sin utilizar métodos restrictivos.

Para iniciar las estrategias, un estudiante del semillero de investigación explicaba las funciones básicas para crear el mundo (figura 6), las propiedades y la forma de insertar los objetos y moverlos, por eso los diseñadores debían seleccionar un objeto e insertarlo, demostrando que a medida que creaban objetos iban agregándole más funcionalidades que permitían un mayor desempeño en el juego. En la tabla 4 se puede ver cómo creaban un objeto (manzana) y a medida que iban creando diferentes copias del mismo objeto empezaban a darle una funcionalidad de acuerdo con el color o tamaño.

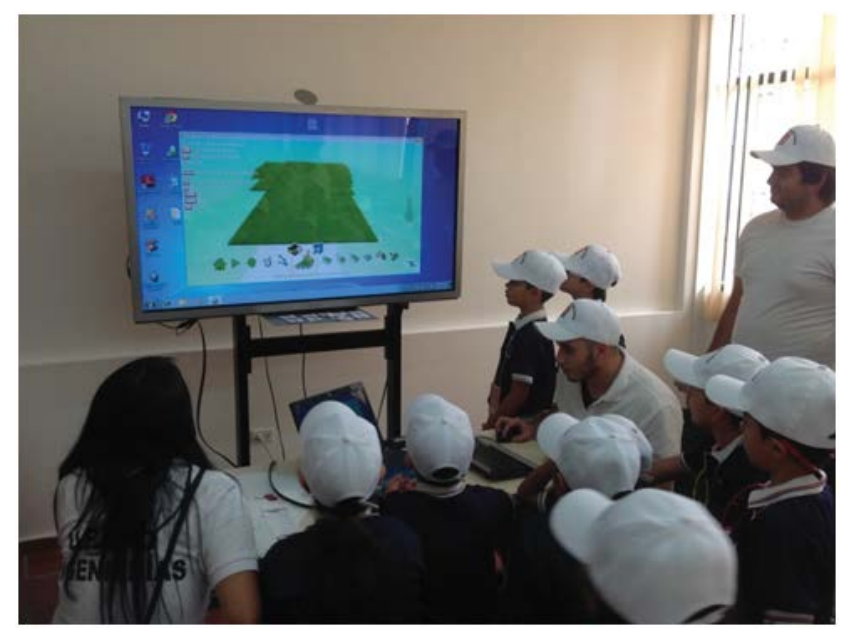

Figura 6. Programación del videojuego.

Fuente: Elaboración propia.

Al principio, el primer objeto se creaba con unas propiedades predefinidas, pero al crear el segundo entendían que podía servir como objeto de alimentación; aquí también empezaban a variar los colores. Ya en el tercer objeto insertaban dos tipos de colores y al objeto rojo lo daban como sistema de alimentación, mientras que al verde como pérdida de puntos, por no estar completamente maduro para su consumo, mientras que el tamaño era extragrande, en el objeto 4 intentaban colocar un obstáculo más fuerte agregándole el color amarillo, el cual podía terminar el juego, un Game

Tabla 4. Funcionalidades en la creación de objetos.

\begin{tabular}{lllll}
\hline & \multicolumn{1}{c}{ Objeto 1 } & \multicolumn{1}{c}{ Objeto 2 } & \multicolumn{1}{c}{ Objeto 3 } & \multicolumn{1}{c}{ Objeto 4 } \\
\hline Color & predefinido & verde & rojo, verde & amarillo \\
\hline Tamaño & predefinido & grande & más grande & grande \\
\hline Funcionalidad & obstáculo & alimentación & pérdida de puntos & envenenamiento \\
\hline Movimiento & No & No & No & No \\
\hline
\end{tabular}

Fuente: Elaboración propia. 
Over por medio y reducían el tamaño de acuerdo a las dimensiones del cachorro y de un objeto como una manzana, esta prueba implicaba que el niño tenía muy claro las funciones de un mundo virtual, las cuales compaginaban con las situaciones de la vida diaria.

En la creación del objeto cachorro, le daban propiedades de un objeto real, de tal forma que empezaban a realizar una programación orientada a objetos sin abstraerse de su mundo y sin conocer las técnicas de programación. Es por eso que al inicio, cuando insertaban el objeto cachorro, tenían claro que podía alimentarse, pero no programaban el movimiento, por lo tanto se volvía un objeto estático; al crear el segundo objeto lo hacían más fuerte al aumentar el tamaño y solo podía alimentarse con objetos rojos, le agregaban movimiento de tal forma que pudiera recorrer todo el mundo. En la creación del objeto tres se vio una clara competencia por superar el reto, ya que el cachorro podía volar, lo que permitía superar los obstáculos y llegar a su destino, sin embargo, en la creación del cuarto objeto entendieron que no podían abstraerse de la realidad y que los perros no volaban (tabla 5), a la vez que infringían las normas del juego, por lo tanto no se sentían transparentes y el tamaño del cachorro no era importante para superar la prueba. Le daban importancia a la alimentación como medio de supervivencia, además pensaron que una fruta no iba a ser lo suficientemente interesante para un cachorro, por lo tanto empezaron a crear distinto objetos que dejaban huellas en la ruta de la vivienda.
Las preguntas finales que podían dar una respuesta sobre el pensamiento científico, investigativo, innovador y creativo de los niños eran:

- ¿Se detectaron niños líderes dentro del proceso?

- ¿El niño buscó diferentes tipos de solución para dar cumplimiento a la actividad?

- ¿Cómo valoraba el niño el trabajo desarrollado por sus compañeros?

- ¿El enfoque del proyecto es el adecuado para el desempeño de los niños dentro del mismo?

- ¿Se identificaron destrezas y conocimientos innatos en los niños para las carreras de ingenierías?

En general, al diseñar el juego con KODU se pudo observar que mantenían su buena disposición al trabajo colaborativo, inclusive a la bondad de sus evaluaciones, ya que un grupo evaluaba a otro grupo (fase VI), y aunque sabían que estaban compitiendo, no intentaban disminuir la nota de sus competidores por el simple deseo de ser el mejor o ganar relevancia. Igualmente tenían un pensamiento crítico, responsable y de recuperación que les permitía corregir los errores, algo que el adulto pierde en el transcurso de los años, como era la posibilidad de hacer trampa., sin embargo sus comités formados por sus cuatro integrantes volvían a tomar acciones que les permitían sentirse seguros de su rol y la función que deben servir en la sociedad.

Entre las habilidades que se pudieron observar en KODU, y algunas preguntas y sus respuestas obtenidas por los docentes y acompañantes del

Tabla 5. Creación del objeto principal.

\begin{tabular}{lllll}
\hline & \multicolumn{1}{c}{ Objeto 1 } & \multicolumn{1}{c}{ Objeto 2 } & \multicolumn{1}{c}{ Objeto 3 } & \multicolumn{1}{c}{ Objeto 4 } \\
\hline Tamaño & predefinido & más grande & Grande & Grande \\
\hline Funcionalidad & $\begin{array}{l}\text { alimentarse con los } \\
\text { objetos }\end{array}$ & $\begin{array}{l}\text { alimentarse solo con } \\
\text { objetos rojos }\end{array}$ & Volar & Correr \\
\hline Movimiento & No & $\mathrm{Si}$ & $\mathrm{Si}$ & $\mathrm{Si}$ \\
\hline
\end{tabular}

Fuente: Elaboración propia. 
proyecto, se encontró que en cada proceso se detectaron líderes naturales, puesto que en muchos casos algunos niños tomaban la iniciativa antes de que se les dieran algunos parámetros. Al utilizar la programación, los niños se convierten en generadores de ideas, por lo tanto en cada actividad siempre plantearon diversas soluciones.

Estar en un escenario diferente ha permitido que los niños valoren el trabajo de sus compañeros, puesto que no están en su ambiente natural; esto despierta en ellos el deseo de apoyarse en todos los procesos, aparte de que fortalecen las ideas de sus compañeros. El enfoque del proyecto está basado en la libertad de desarrollar las actividades sin imposiciones, de acuerdo con sus gustos, necesidades y, sobre todo, está orientado a culminar cada actividad con las soluciones que ellos han generado.

Las ingenierías son carreras con la capacidad de desarrollar procesos creativos, y esto tiene un atractivo especial para los niños, porque en la actualidad nacen con destrezas innatas para el manejo de computadores, videojuegos, etc.; les encanta todo aquello en lo cual puedan crear sus propias cosas de acuerdo con sus intereses. En el momento de desarrollar el proyecto con la carrera de ingeniería de sistemas, también han empezado a trabajar con la carrera de ingeniería civil, mediante la creación de edificios y estructuras, y sobre todo ver que para los niños un computador forma parte de su quehacer, este mismo software permitió en los niños plantear algunos retos de tal forma que se pueda llevar a cabo en las carreras de Contaduría Pública y Comunicación Social, y permita la creación de sus propios mundos virtuales, después de conocer todos los roles de dichas carreras.

\section{CONCLUSIONES}

Con la realización del proyecto Universidad de los Niños, para este caso niño Ingeniero de Sistemas, se puede concluir que los niños son innovadores por naturaleza y tienen la capacidad de dar soluciones con la facilidad que un adulto ha perdido; se pudieron determinar estas destrezas a través del software KODU, en el cual los niños identifican, a través del videojuego, las mejores opciones para dar solución al problema planteado.

Los rígidos modelos que han presenciado los niños a través de su vida escolar crean un adolescente que ha disminuido la motivación, creatividad, innovación e investigación propia de la carrera de Ingeniería de Sistemas; por tal motivo, la Universidad de los Niños, muestra que la ingeniería de sistemas es una carrera ideal para rescatar lo innato de su ser.

\section{FINANCIAMIENTO}

Del proyecto titulado "Universidad de los niños - Niño Ingeniero de Sistemas", Universidad Francisco de Paula Santander Ocaña, por sus recursos físicos y condiciones para garantizar la investigación.

\section{REFERENCIAS}

Barrionuevo, A. (2009). Lenguajes de Programación para niños. Revista Digital Enfoques Educativos (51), 29-39.

Buchsbaum, D., Gopnik, A., \& Griffiths, T. L. (2010). Children's Imitation of Action Sequences is Influenced by Statistical Evidence and Inferred Causal Structure. Proc. of the 32nd Annual Conference of the Cognitive Science Society .

Cacia, B., Chiazzese, G., \& D' Amicoa, A. (2013). Robotic and Virtual World Programming labs to Stimulate Reasoning and visual-spatial Abilities. 93, 1493-1497.

Gallego, A. P., Castro, J., \& Rey, J. (2008). El pensamiento Científico en los niños y las niñas: algunas consideraciones e implicaciones. CIEEC , 2 (3), 22-29.

Kushnir, T., Xu, F., \& Wellman, H. (2010). Young children use statistical sampling to infer the preferences of others. NIH Public Acces , 21 (8), 1134-1140. 
Lucas, C., Bridgers, S., Griffithsc, T., \& Gopnik, A. (2014). When children are better (or at least more open minded) learners than adults: Developmental defferences in learning the forms of causal relationships. International Journal of Cognitive Science, 131 (2), 284-299.

Lukas, C., Griffiths, T., Fawcett, C., \& Gopnik, A. (2014). The child as Economietrician: A Rational Model of Preference Understanding in Children. PLOS ONE, 9 (3).

Ma, L., \& Xu, F. (2014). Young children's use of statistical sampling evidence to infer the subjectivity of preferences. International Journal of Cognitive Science, 120 (3), 403-411.
Merzagora, M., \& Jenkins, T. (2013). Listening and Empowering Children in Science Communication. Jcom Journal of Science Communication, 12 (3).

Miraut, D., \& Mendoza, A. (2011). Iniciativas educativas para una sociedad de la información sostenible, Teoría de la Educación. Educación y Cultura en la Sociedad de la Información , 12 (3), 241-264.

Trujillo, E. (2001). Desarrollo de la actitud científica en niños de edad preescolar. Anales de la Universidad Metropolitana, 1 (2), 187-195. 